\title{
Exploration of the surface director profile in a liquid crystal cell using coupling between the surface plasmon and half-leaky optical guided modes
}

\author{
Fuzi Yang, ${ }^{1, a)}$ Lizhen Ruan, $^{2}$ and J. R. Sambles ${ }^{2}$ \\ ${ }^{1}$ Chemistry Department, Tsinghua University, Beijing 100084, People's Republic of China \\ ${ }^{2}$ Electro-Magnetic Materials, School of Physics, University of Exeter, Exeter EX4 4QL, United Kingdom
}

(Received 21 January 2008; accepted 24 March 2008; published online 15 April 2008)

\begin{abstract}
The half-leaky guided mode geometry with a thin metal tunnel barrier as one cladding layer is used to explore the distribution of the director very near to the surface in a hybrid aligning nematic liquid crystal cell. From theoretical analysis together with numerical modeling, it is shown how the coupled $p$-polarized surface plasmon/s-like guided modes excited in the geometry leads to extremely sensitive to the surface director tilt profile near the metal wall-a sensitivity which is even higher than that of the surface plasmon resonance alone. The experimental results have fully confirmed the model predictions. (C) 2008 American Institute of Physics. [DOI: 10.1063/1.2908224]
\end{abstract}

Some early studies have explored the surface plasmonpolariton (SPP) mode at the interface between a silver layer and a nematic liquid crystal (LC) to probe the phase transition and the voltage dependence of the director tilt at the wall of the LC cell. ${ }^{1-3}$ These studies examined only the SPP mode, even though optical guided modes should also have been found in some sample. Later, Sambles and co-workers ${ }^{4,5}$ examined both optical guided modes and SPP in silver-clad nematic LC cells. They thereby obtained information about both near-surface and in-the-bulk director profiles at the same time.

In a continuation of these studies, Elston and Sambles ${ }^{6,7}$ explored the director profile of a surface stabilized ferroelectric LC cell for which the optical mode spectrum was far richer, involving the excitation of SPP and two types of guided modes associated with the low index and high indices of the LC, respectively. These studies appear to be some of the earliest to show that the SPP may couple with optical guided modes through polarization conversion due to the director geometry.

By using a hybrid aligned nematic LC (HAN-LC) cell symmetrically clad with silver films we have presented a study $^{8}$ exploring this optical coupling between the SPP and guided modes in much more detail and shows that these couplings are very sensitive to the details of the near-surface director profile.

In this present, study the half-leaky guided mode (HLGM) geometry ${ }^{9}$ with a thin metal tunnel barrier as one cladding layer is used. Both theoretical analysis and numerical modeling show that the coupling spectrum between the SPP and the optical guided modes excited in this HAN-LC geometry can also give a high sensitivity to the surface director tilt profile near the metal layer-a sensitivity which is higher than that of the surface plasmon mode alone. Experimental results fully confirm our model predictions.

The model of HLGM-HAN-LC cell is shown in the inset of Fig. 1. As shown in Fig. 1, the director of the LC lying in plane zox (having twist angle $\varphi \equiv 0^{\circ}$ ), for a $p$-polarized incident beam in plane zoy a SPP is excited having in-plane ( $y$ direction) momentum which is mainly dependent upon an effective index $n_{p}$ along the $z$ direction. In the region close to the upper (metal) boundary, the tilt angle is

\footnotetext{
${ }^{a)}$ Electronic mail: f.yang@exeter.ac.uk.
}

closely $\theta=90^{\circ}$ and linearly varies with depth $t$ away from the top wall by a quite small gradient, $\delta \theta^{\prime}=d \theta / \mathrm{dt} \ll 1$. Thus, if the ordinary and extraordinary refractive indices of the LC are $n_{o}$ and $n_{e}$, then in this top area the $z$ direction effective refractive index at depth $t$ is

$$
n_{\mathrm{pt}}=\frac{n_{o} n_{e}}{\sqrt{n_{e}^{2} \cos ^{2}\left(\delta \theta^{\prime} t\right)^{2}}+n_{o}^{2} \sin ^{2}\left(\delta \theta^{\prime} t\right)^{2}} .
$$

Because $\delta \theta^{\prime} t \ll 1$ in the upper thin region, i.e., $n_{\mathrm{pt}} \sim n_{o}$, we can still consider that the SPP mode is excited on the interface between a metal and a dielectric with a $z$ direction refractive index $\bar{n}_{\mathrm{p} t}$, which is the average of $n_{\mathrm{pt}}$ over the SPP penetration depth $t_{\mathrm{SPP}},{ }^{10}$

$$
t_{\mathrm{SPP}}=\frac{\lambda}{2 \pi} \sqrt{\frac{\varepsilon_{d}+\left|\varepsilon_{m}^{\prime}\right|}{\varepsilon_{d}^{2}}},
$$

where $\varepsilon_{d}$ and $\varepsilon_{m}^{\prime}$ are the permittivity of the dielectric and real part of the permittivity of the metal, respectively. Thus, we should have

$$
\bar{n}_{\mathrm{pt}}=\int_{0}^{t_{\mathrm{SPP}}} n_{\mathrm{pt}} d t / t_{\mathrm{SPP}}=n_{o}+\Delta n,
$$

with

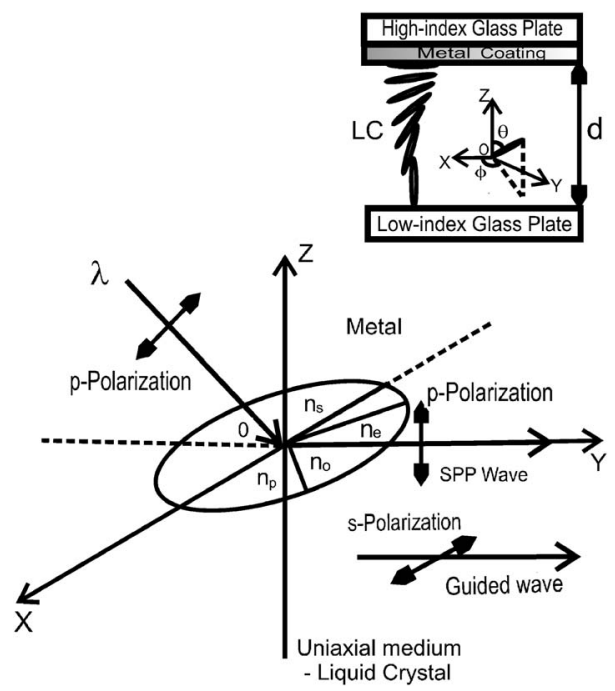

FIG. 1. The SPP mode and guided waves at the top-boundary area of a HAN cell. The inset is a HLGM-HAN-LC cell with a metal tunnel barrier. 

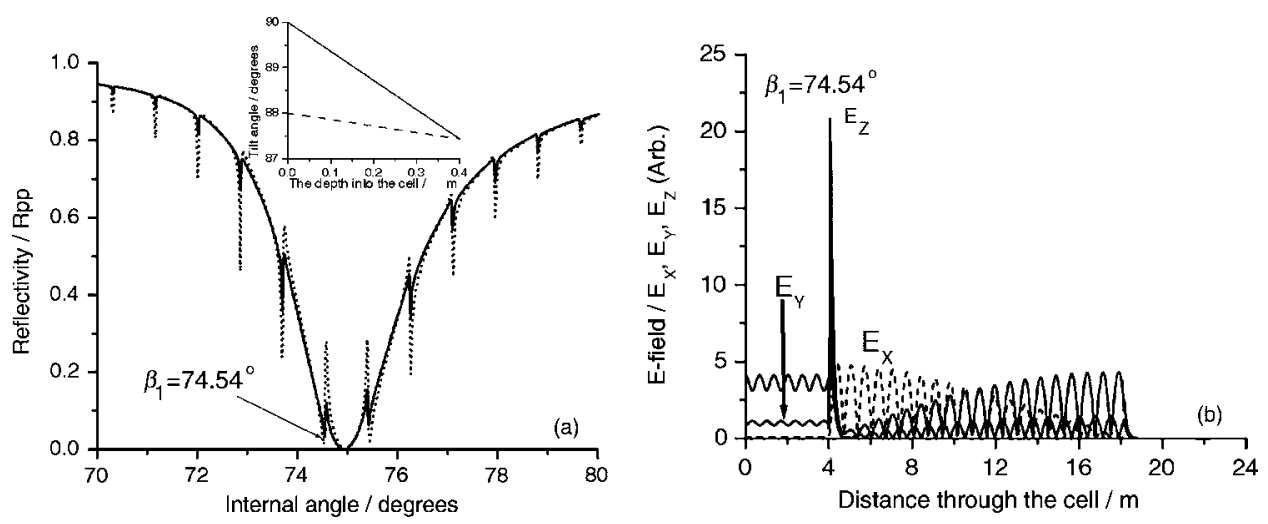

FIG. 2. (a) The model results of the reflectivity $\left(R_{\mathrm{pp}}\right)$ in the SPP mode area for two different tilt angle profiles, which are shown in the inset. The parameters for the modeling are $\lambda=632.8 \mathrm{~nm}$, for the top glass plate $\varepsilon_{H}=2.9998$, for thin silver film $\varepsilon_{\mathrm{Ag}}=-17.50+i 0.65$ with thickness $d_{\mathrm{Ag}}=50 \mathrm{~nm}$, for the SiO ${ }_{x}$ layer $\varepsilon_{s}=2.665+i 0.002$ with thickness $d_{s}=30 \mathrm{~nm}$, for LC $\varepsilon_{x x}=\varepsilon_{y y}=2.3132+i 0.0002$ and $\varepsilon_{z z}=3.0145+i 0.0002$ with a thickness $d_{\mathrm{LC}}=14.00 \mu \mathrm{m}$ and $\theta 1=90.0^{\circ}$ at the top and $\theta_{2}=0.0^{\circ}$ at the bottom, $\varphi=0^{\circ}$ through the cell, for the bottom glass plate $\varepsilon_{L}=2.1418$, for the ITO coating $\varepsilon_{I}=3.000+i 0.02$ with thickness $d_{I}=75 \mathrm{~nm}$. The very thin lecithin layer can be ignored as far as its optical effect is concerned. (b) The optical $E$-field distribution at the incident angle $\beta_{1}=74.54^{\circ}$.

$$
\Delta n=\frac{\lambda^{2}}{24 \pi^{2} n_{o}^{3}}\left(1-n_{o}^{2} / n_{e}^{2}\right)\left(n_{o}^{2}+\left|\varepsilon_{m}^{\prime}\right|\right)\left(\delta \theta^{\prime}\right)^{2} .
$$

This $\bar{n}_{\mathrm{pt}}$ determines the wave vector of the SPP mode. For a cell with a thickness $d=12 \mu \mathrm{m}$ and $n_{o}=1.517, n_{e}=1.732$ for LC, $\varepsilon^{\prime}{ }_{m}=-18.0$ for silver at $\lambda=632.8 \mathrm{~nm}$, then with no externally applied voltage $\delta \theta^{\prime}=\pi / 24=0.1309 / \mu \mathrm{m}$ and $\Delta n$ $=0.00004$, which is a very small change. Even for $\delta \theta^{\prime}$ $=0.292 / \mu \mathrm{m}$, which corresponds to an applied voltage of about $1.0 \mathrm{~V}, \Delta n$ is still only $\sim 0.0002$. It is thus apparent that the SPP alone does not readily distinguish the difference of such a small tilt angle deviation at the metal boundary.

The SPP will be the highest in-plane momentum transverse magnetic (TM) mode for a relatively thick HAN cell under no or low externally applied voltage. ${ }^{8}$ However, for the HLGM geometry at an incident angle range, ${ }^{9}$ some $s$-type guided modes may also be excited by the $p$ to $s$ conversion in the upper wall area. The maximum in-plane momentum of $s$-type guided modes is mainly dependent upon the effective index $n_{s}$ (also see Fig. 1). Close to the upper wall $\theta \approx 90^{\circ}$ and thus $n_{s} \approx n_{e}$. Therefore, for a positive LC $\left(n_{e}>n_{o}\right)$, there is the possibility of $s$-type guided waves appearing in the angle range of the SPP resonance. Even though the intensity ratio of this $p-s$ conversion is only proportional to $\sin ^{2}\left(\delta \theta^{\prime} t\right) \cos ^{2}\left(\delta \theta^{\prime} t\right) \sim\left(\delta \theta^{\prime} t\right)^{2}$ and very small, however, from the excitation of the SPP mode the maximum field enhancement at the boundary between the metal and the low index dielectric (see the inset of Fig. 1) should be ${ }^{11}$

$$
T_{\max }^{e l}=\frac{2\left|\varepsilon_{m}^{\prime}\right|^{2}\left[\left|\varepsilon_{m}^{\prime}\right|\left(\varepsilon_{H}-1\right)-\varepsilon_{H}\right]^{1 / 2}}{n_{o}^{2} \varepsilon_{m}^{\prime \prime}\left(1+\left|\varepsilon_{m}^{\prime}\right|\right)} .
$$

If $n_{o}=1.517, n_{H}=1.732, \varepsilon_{m}^{\prime}=-18.0$, and $\varepsilon_{m}^{\prime \prime}=0.65(\mathrm{Ag}$ at $632.8 \mathrm{~nm}$ ), then $T_{\max }^{e l} \approx 130$. Thus, the average field enhancement from the boundary to the penetration depth $t_{\mathrm{SPP}}$ is about 82. Given that the power enhancement scales as this factor squared then significant signatures may appear in the SPP resonance. Thus, we expect the coupling spectrum between the $p$-polarized SPP resonance and the $s$-polarized guided modes to be particularly sensitive to the near-surface director tilt profile.

We have used the Berreman $4 \times 4$ matrix to model a HLGM-HAN cell with geometry of high index glass platesilver coating $60^{\circ}$ evaporated $\mathrm{SiO}_{x}$ layer (for homogeneously lver coating $60^{\circ}$ evaporated $\mathrm{SiO}_{x}$ layer (for homogeneously and $1.00 \mathrm{~V}$, respectively.
Downloaded 20 Jan 2009 to 144.173 .6 .75 . Redistribution subject to AlP license or copyright; se aligning) HAN LC layer-lecithin layer (for homeotropicaly aligning) indium tin oxide (ITO) coating low index glass plate. The parameters of the geometry are given in the caption of Fig. 2. In the modeling, two tilt profiles have been chosen. In the first the tilt angle linearly varies from $90^{\circ}$ to $0^{\circ}$ across the whole cell, for the second one the tilt linearly varies from $88^{\circ}$ to $87.43^{\circ}$ in the area close to the upper boundary up to $0.40 \mu \mathrm{m}$ and then it remains the same as the first profile through the rest of the cell, modeling in effect something like weak anchoring at the top surface. These two tilt angle profiles at the top surface are shown in the inset of Fig. 2(a) as solid and dashed lines, respectively. Two $R_{\mathrm{pp}}$ curves, have been computed and shown in Fig. 2(a) as solid and dotted lines, respectively. It is obvious that the coupling spectra between the SPP resonance and the $s$-type guided modes show significant differences between these two surface tilt distributions, even though the envelope of the SPP shows no change. At the incident angle $\beta_{1}=74.54^{\circ}$ shown in Fig. 2(a) by an arrow, at which the coupling of the two types of mode occurs, the optical field distribution through the cell has been modeled and shown in Fig. 2(b). It is very clear that the sharp "hole" in the SPP resonance arises from the coupling between the SPP and guided modes, as shown by the TM-TE field distribution through the cell and the TM field enhancement (SPP) at the interface.

To confirm the modeling by experiment, a sample cell with the geometry mentioned above has been built. Two mylar strips with thickness about $12.0 \mu \mathrm{m}$ are used as the cell gap spacers and the cell is capillary filled with a nematic LC (E7, Merck-BDH) at room temperature. High indexmatching fluid is used to optically match the high index glass prism to the high index top plate of the cell. The experimental setup is a typical $\theta-2 \theta$ rotating system as described elsewhere. ${ }^{9}$ A $p$-polarized beam with $\lambda=632.8 \mathrm{~nm}(\mathrm{He}-\mathrm{Ne})$ incomes on one face of the prism. Then the incident angle dependent reflectivity signals leaving from a second side face of the prism are recorded using a detector with a $p$-polarizer placed in front of it. Data were taken with voltages applied to the cell of $0.00,0.50,0.75$, and $1.00 \mathrm{~V}(\mathrm{rms})$, at a frequency of $10 \mathrm{kHz}$. Two typical $R_{\mathrm{pp}}$ signals are shown in Figs. 3 and 4 as the circles for the applied voltages of 0.00

o AIP license or copyright; see http://apl.aip.org/apl/copyright.jsp 


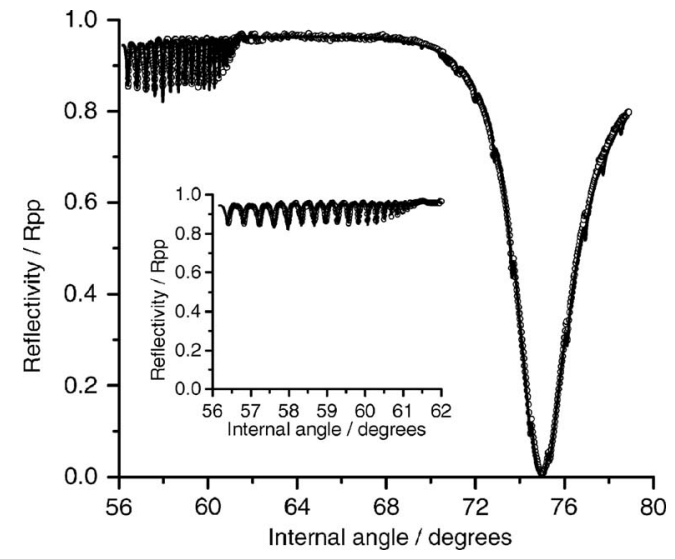

FIG. 3. The whole incident angle range of the reflectivity $R_{\mathrm{pp}}$ data (circles) and fitting result (solid line) for no voltage applied. The inset is the detail of fitting results and experimental data in the TM-type guided mode region. The parameters of the sample geometry from the fit are: silver film $\varepsilon_{\mathrm{Ag}}=-17.41+i 0.69, d_{\mathrm{Ag}}=49.0 \mathrm{~nm} ; \quad \mathrm{LC}$ (E7) $\varepsilon_{x x}=\varepsilon_{y y}=2.3132+i 0.0003$, $\varepsilon_{z z}=3.0145+i 0.0003, d_{\mathrm{LC}}=14.65 \mu \mathrm{m}$ with twist angle $\varphi=0.0^{\circ}$ (out-of-plane geometry); $\mathrm{SiO}_{x}$ and ITO are same as in Figure 2(a).

Two typical fits for 0.0 and $1.00 \mathrm{~V}$ are also shown in Figs. 3 and 4 as the solid lines. The fitting of the $0 \mathrm{~V}$ data over a wider angle range, including TM-like guided waves and the SPP mode, is very important for determining the basic parameters of the geometry. Except for the known the permittivities of 2.9998 and 2.1418 for the glass plates, the parameters in the sample geometry from the fits are shown in the caption of Fig. 3. Continuum elastic theory ${ }^{12}$ gave the director profile using elastic constants $K_{11}=11.42 \mathrm{pN}, K_{33}$ $=18.20 \mathrm{pN}$, and both tilt anchoring strengths of $W_{\theta 1}=2.5$ $\times 10^{-4} \mathrm{~J} / \mathrm{m}^{2}$ and $W_{\theta 2}=2.0 \times 10^{-3} \mathrm{~J} / \mathrm{m}^{2}$ on the top and bottom boundaries. Details of the experimental data (circles) and theoretical fit (solid line) to the TM-type guided mode region are shown in the inset of Fig. 3. For the case with applied voltages the anisotropic dielectric permittivities are chosen as $\varepsilon_{\|}=19.50$ and $\varepsilon_{\perp}=5.40$ for the E7 at room temperature. The solid line in Fig. 4 is the typical fitting result for an applied voltage of $1.00 \mathrm{~V}$ with the tilt angle profile through the cell, which is modeled from the continuum elastic theory and used to calculate the fitting result. In the inset of Fig. 4 details of the tilt angle variations at the upper boundary area for the case of no applied voltage and $1.00 \mathrm{~V}$ are shown as dotted and solid lines, respectively. From the inset, it is clear that the surface tilt angle deviates from horizontal $\left(\theta_{1}=90^{\circ}\right)$ due to the balance between surface anchoring, elastic forces and E-field coupling to the director. If the surface anchoring force at the upper surface is not strong enough, thereby letting the surface tilt angle change to $86.0^{\circ}$, then the tilt profile shown as the dash-dotted line in the inset with no change in the remainder of the cell may be imagined. Even though the difference between the two cases is quite small, the coupling spectrum still distinguishes them, as shown in Fig. 4 in which the dotted line, which is predicted from the imagined surface tilt profile shown in the inset as a dash-dotted line. However, it is clear that the envelope of the SPP mode does not change at all for both cases. This not only demonstrates that the coupling spectrum is very sensitive to the surface director profile, but also indicates that the surface tilt angle profile predicted by the continuum elastic theory ${ }^{12}$ is reasonable.

In conclusion, theoretical analysis and numerical modeling have shown that for a HLGM-HAN-LC cell with a silver

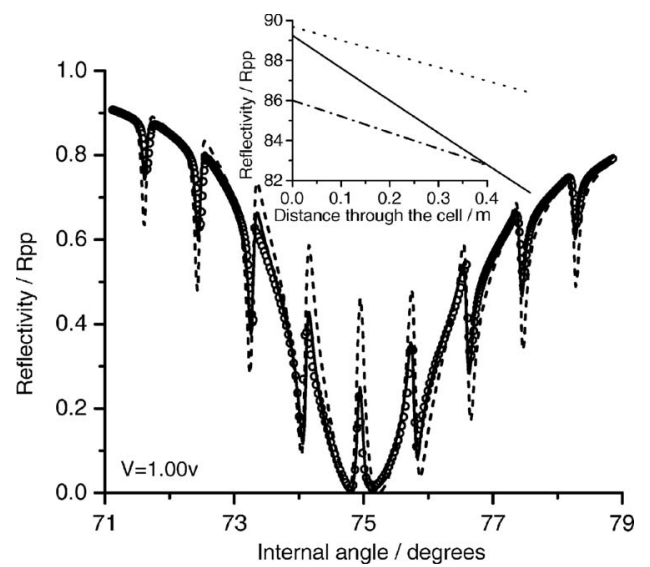

FIG. 4. The reflectivity $R_{\mathrm{pp}}$ data (circles) and model fit (solid) at $1.00 \mathrm{~V}$. The dotted line is the model coupling spectrum for the tilt angle distribution shown in the inset by the dash-dotted line. The inset is the detail of the tilt angle distributions near the upper boundary. The solid line and dotted lines are for applied voltage of 1.00 and $0 \mathrm{~V}$, respectively, while the dash-dotted line is an imaginary profile with the surface tilt angle set at $86^{\circ}$.

tunnel barrier and $p$-polarized incident beam the polarization coupling spectrum between $s$-type guided waves and the $p$-polarized SPP mode is a very sensitive probe for exploring the director profile near the wall area. By using this spectrum very small differences in the director profile near the wall area can be distinguished, even though the surface plasmon mode shows no significant changes.

Experimental results from a LC cell mentioned above have firmly confirmed the analytical and modeling predictions show clearly how the top-surface director profile varies with low external applied voltages. Because the low-index plate, for a certain incident angle range, is a better total reflector than the metal-coating used in the symmetric geometry $^{8}$ then not only is the coupling spectrum excited, but it also means it is somewhat easier to fabricate the sample. Further there are less optical parameters in the fitting procedure making that easier while the coupling effect appears somewhat stronger because of the lack of the bottom metal layer. This work can readily be extended to studying the dynamic behavior ${ }^{13}$ of the surface director, and it should therefore prove to be a very powerful tool for surface LC physics research.

Fuzi Yang appreciates the support of the University of Exeter and Dr. Lizhen Ruan acknowledges the support provided by EPSRC.

${ }^{1}$ K. C. Chu and C. K. Chen, Mol. Cryst. Liq. Cryst. 59, 97 (1980).

${ }^{2}$ N.-M. Chao, K. C. Chu, and Y. R. Shen, Mol. Cryst. Liq. Cryst. 67, 261 (1981).

${ }^{3}$ G. J. Sprokel, R. Santo, and J. D. Swalen, Mol. Cryst. Liq. Cryst. 68, 29 (1981).

${ }^{4}$ K. R. Welford and J. R. Sambles, Appl. Phys. Lett. 50, 871 (1987).

${ }^{5}$ K. R. Welford, J. R. Sambles, and M. G. Clark, Liq. Cryst. 2, 91 (1987).

${ }^{6}$ S. J. Elston, J. R. Sambles, and M. G. Clark, J. Mod. Opt. 36, 1019 (1989).

${ }^{7}$ S. J. Elston and J. R. Sambles, Jpn. J. Appl. Phys., Part 2 29, L641 (1990).

${ }^{8}$ F. Yang, L. Z. Ruan, S. A. Jewell, and J. R. Sambles, New J. Phys. 9, 49 (2007).

${ }^{9}$ F. Yang and J. R. Sambles, J. Opt. Soc. Am. B 10, 858 (1993).

${ }^{10}$ H. Raether, Surface Plasmons (Springer, Berlin, 1988).

${ }^{11}$ H. Raether, Surf. Sci. 140, 31 (1984).

${ }^{12}$ F. M. Leslie, Mol. Cryst. Liq. Cryst. 12, 57 (1970).

${ }^{13}$ F. Yang, Y. Dong, L. Z. Ruan, and J. R. Sambles, J. Appl. Phys. 96, 310 (2004). 